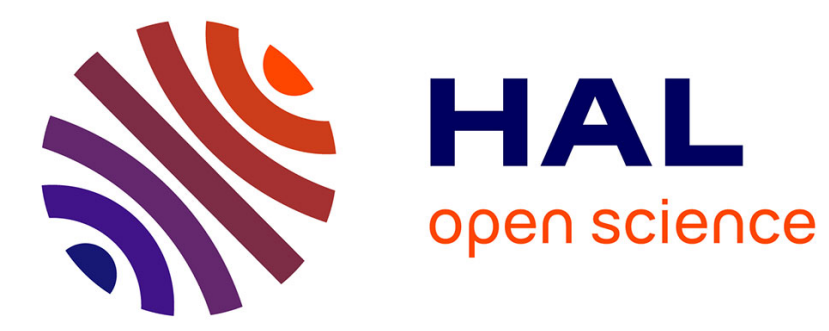

\title{
Interactive leader-follower consensus of multiple quadrotors based on composite nonlinear feedback control
}

\author{
Zhicheng Hou, Isabelle Fantoni
}

\section{- To cite this version:}

Zhicheng Hou, Isabelle Fantoni. Interactive leader-follower consensus of multiple quadrotors based on composite nonlinear feedback control. IEEE Transactions on Control Systems Technology, 2018, 26 (5), pp.1732-1743. 10.1109/TCST.2017.2738602 . hal-01635281

\section{HAL Id: hal-01635281 \\ https://hal.science/hal-01635281}

Submitted on 14 Nov 2017

HAL is a multi-disciplinary open access archive for the deposit and dissemination of scientific research documents, whether they are published or not. The documents may come from teaching and research institutions in France or abroad, or from public or private research centers.
L'archive ouverte pluridisciplinaire HAL, est destinée au dépôt et à la diffusion de documents scientifiques de niveau recherche, publiés ou non, émanant des établissements d'enseignement et de recherche français ou étrangers, des laboratoires publics ou privés. 


\title{
Interactive leader-follower consensus of multiple quadrotors based on composite nonlinear feedback control
}

\author{
Zhicheng Hou, Isabelle Fantoni, Member, IEEE
}

\begin{abstract}
In this paper, an interactive L-F configuration of multiple quadrotors is considered, which means that the leaders can react with respect to the followers. In order to analyze the consensus of such a multi-quadrotor system, an interaction matrix is proposed. The convergence condition of the trajectory tracking error is then proven to be related to the smallest eigenvalue of the interaction matrix. In order to achieve an aggressive formation, we develop a bounded composite nonlinear feedback based formation controller with hyperbolic functions as a main contribution. Simulation and real-time experimental results show that the formation task is achieved with small overshoot and rapid response speed. Real-time experiments show four quadrotors following a circular reference trajectory with high linear velocity up to $2.7 \mathrm{~m} / \mathrm{s}$.
\end{abstract}

Index Terms-quadrotors, consensus, leader-follower, aggressive formation, bounded composite nonlinear feedback control

\section{INTRODUCTION}

$\mathbf{T}$ HE natural behavior of animals operating as a team has inspired scientists in different disciplines to investigate the possibilities of networking a group of systems to accomplish a given set of tasks without requiring an explicit supervisor [1]. Therefore, multi-agent systems have broadly appeared in several applications including multi-vehicle system [2] [3], formation flight of unmanned air vehicles (UAVs) [4] [5] [6], sensor networks [7], self-organization, automated highway systems, and congestion control in communication networks [8].

The guidance of quadrotors with a formation pattern to track a given trajectory has potential applications. Researchers in [9] develop a tele-operation strategy for multi-robot system. Within this scope, they investigate the strategies such as selection of leaders in the group of mobile robots, in order to achieve some optimization goals. Researchers in [10] investigate the connectivity maintenance problem in multiquadrotor systems. They have proposed a method to maintain the topology graph connected through changing the weights in adjacency matrix. They use the second smallest eigenvalue

This work was carried out and funded in the framework of the Labex MS2T (Reference ANR-11-IDEX-0004-02) and the ROBOTEX Equipment of Excellence (Reference ANR-10-EQPX-44). They were supported by the French Government, through the program Investments for the future managed by the National Agency for Research.

Zhicheng Hou is with Guangzhou Institute of Advanced Technology, Chinese Academy of Sciences, China. zc.houlgiat.ac. cn

Isabelle Fantoni is with Sorbonne universités, Université de technologie de Compiègne, CNRS, UMR 7253 Heudiasyc, 60200 Compiègne, France. isabelle.fantoniehds.utc.fr of the Laplacian as a measurement of the graph connectivity. Then, they develop a controller to increase this eigenvalue, which is estimated with a decentralized method.

The usage of multiple quadrotors to transport large payloads is considered in [11], [12] and [13]. The flexible payload transportation using the cooperation of multiple quadrotors is investigated [6], where the control with prediction is proposed to reduce the deformation of the payload. The applications such as search and rescue are covered in [14]. The problem of cooperative surveillance in large outdoor areas by a fleet of micro aerial vehicles is studied in [15]. The visibility maintenance problem of the multi-robot system with obstacle environments are investigated through using vision [16]. The aggressive formation of quadrotors is considered in [17], where the leader-follower configuration is used. However, in order to deal with the delay of the communication over network, the leading robot computes its desired trajectory and broadcasts to all follower robots a message containing the polynomial coefficients and time intervals that fully specify its trajectory. Cooperative quadrotors are also used for architecture [18].

In our work, the condition of connectivity is not necessarily needed, we just need a positive-definite interaction matrix. Furthermore, a leader's states can only be used by its neighboring quadrotors instead of all the quadrotors. Our main goal is here to propose an efficient controller, which contains a rapid rising response and small overshoot during aggressive manoeuvers, as it will be explained below.

The use of UAVs able to maintain hovering flight for surveillance implies that the power of actuators is sufficient. In practice, the motors of a quadrotor have a limited power. Therefore, the inputs of a quadrotor are subject to actuator saturations. The saturated control is progressively implemented on quadrotors in order to prevent the control signal from hitting the actuator saturations. For example, authors in [19] introduce a nested saturation controller on PVTOL aircraft, which globally stabilizes the PVTOL aircraft around the origin $((x, y, \theta) \equiv(0,0,0))$. The design of the nested saturation controller is based on the linearization around the origin by calculating the Jacobian matrix. In [20], the nested saturation controller is proposed to generate bounded inner-loop inputs, assuming that the inner-loop dynamics can perfectly track its input. However, in these two cases, the use of standard saturation functions can cause control problems, because the standard saturation functions have non-differentiable points. Furthermore, the controller design is based on the linearization 
of the nonlinear dynamics. The linearization may probably make the performance of the quadrotor getting worse when the quadrotor behaves aggressively. In [21], the authors propose an algorithm of feasible trajectories planning, considering the input constraints of the quadrotors. This method has limits on the given trajectories, which sometimes dissatisfy the practical requirement.

To the best of our knowledge, the input saturations are not frequently considered in the multi-quadrotor systems control. In [22], a low gain feedback control is proposed to avoid the saturation of the system, such that a semi-global leaderfollower consensus of linear multi-agent system is achieved. In the formation control of multi-UAV systems, a large formation error can generate a large control output. This could lead to big attitude angles for the UAVs of the formation. The low gain controller [22] can guarantee the control output to be small, however, the response speed of the closed-loop system is low.

In recent years, authors in [23] [24] have proposed and implemented a "composite nonlinear feedback" (CNF) control to improve the performance of a system. The objective of this control method is to reduce the overshoot and meanwhile, keep a rapid rising response. We have been inspired by the $\mathrm{CNF}$ approach in our proposed strategy.

In this paper, a new type of leader-follower formation is considered, where the leader(s) has interactions with the followers. Only part of the followers can sense the leader(s). A UAV, although the leader, interacts with its neighbors instead of all the agents. Among the quadrotors, the one who uses the states of the reference formation trajectory in its controller is a leader. The consensus is analyzed in terms of a proposed "interaction matrix" instead of a "Laplacian". The connectivity of the graph is not necessarily required, while we only need to show that the interaction matrix is invertible.

Based on added saturation control, we develop a bounded formation controller with CNF. We replace the standard saturation function by the hyperbolic tangent function, which is always derivable w.r.t time. Furthermore, a composite nonlinear feedback is added in order to improve the formation performance in transient period. We detail the procedure of the $\mathrm{CNF}$ for the consensus of multiple quadrotors and implement the controller in simulations and in real-time on four quadrotors with a satisfactory performance. The formation control strategy is designed to achieve a fixed formation pattern while accomplish an aggressive trajectory tracking of the formation. According to [25], the aggressive flight means that a quadrotor flight in regimes beyond hover conditions. The experimental results in [25] show that the pitch and roll angles can be up to \pm 15 degrees. In this paper, the aggressive flight of quadrotors in formations can fly with pitch and roll angles greater than 5 degrees but less than 30 degrees. The investigated bounded formation controller is differentiable and makes the closedloop system to have fast response speed and small overshoot.

The paper is organized as follows. Some preliminaries are shown in section [II The consensus control of a formation of quadrotors are presented in section III Simulation and experimental results are given in section IV] Finally, some conclusions are stated in section $\mathrm{V}$

\section{PRELIMINARIES}

\section{A. Interaction matrix}

In multi-agent systems, the interaction topologies of agents are represented using a graph $\mathcal{G}=(\mathcal{V}, \mathcal{E})$ with the sets of vertices $\mathcal{V}$ and edges $\mathcal{E}$. The set of vertices $\mathcal{V}=\{1,2, \ldots, n\}$ is composed of the indices of agents. $|\mathcal{V}|$ represents the cardinality of the set $\mathcal{V}$, which satisfies $|\mathcal{V}|=n$. The set of edges is represented by $\mathcal{E} \subseteq \mathcal{V} \times \mathcal{V}$. If an edge exists between two vertices, the two vertices are called adjacent. A graph is simple if it has no self-loops or repeated edges. In other words, the edge $(i, i)$ does not exist. The graph $\mathcal{G}$ is said to be undirected if $(i, j) \in \mathcal{E} \Leftrightarrow(j, i) \in \mathcal{E}$. In this work, simple and undirected graphs are considered.

A path between two vertices $i, j$ is a sequence of edges in a graph of the form $\left(i, i_{1}\right),\left(i_{1}, i_{2}\right), \ldots,\left(i_{k}, j\right)$. A graph $\mathcal{G}$ is connected if there is a path between any two vertices, otherwise it is disconnected. The adjacency matrix of $\mathcal{G}$ is denoted by $G^{A}=\left[\omega_{i j}^{a}\right] \in R^{n \times n}$, where $\omega_{i j}^{a}$ represents the entry on the $i$ th row $j$ th column of matrix $G^{A}$. Since the simple graph is considered, we have $\omega_{i i}^{a}=0$. Since the graph is undirected, we have $\omega_{i j}^{a}=\omega_{j i}^{a}$ and $\omega_{i j}^{a}=1$ if $(i, j) \in \mathcal{E}$, otherwise, $\omega_{i j}^{a}=0$. The degree matrix of $\mathcal{G}$ is denoted by $G^{D}=\operatorname{diag}\left\{\sum_{j=1}^{n} \omega_{1 j}^{a}, \ldots, \sum_{j=1}^{n} \omega_{n j}^{a}\right\}$.

The neighbor set $\mathcal{N}_{i}=\{j \in \mathcal{V}:(i, j) \in \mathcal{E}\}$ of agent $i$, is composed of the indices of the agents $j$, which has interaction with the agent $i$. In other words, if $\omega_{i j}^{a}>0$, then, agent $j$ is a neighbor of agent $i$. The number of the neighbors of the agent $i$ is equal to $\left|\mathcal{N}_{i}\right|$.

We also define a diagonal matrix $G^{L}=\operatorname{diag}\left\{\omega_{1}^{l}, \ldots, \omega_{n}^{l}\right\}$ representing the status of agents. If $\omega_{i}^{l}=1$, then agent $i$ is a leader. Otherwise, if $\omega_{i}^{l}=0$, agent $i$ is a follower, for $i \in \mathcal{V}$. Then, the leader set is defined as $\mathcal{V}_{L}=\left\{i \in \mathcal{V}: \omega_{i}^{l}>0\right\}$. The leader set $\mathcal{V}_{L} \subset \mathcal{V}$ is a subset of $\mathcal{V}$, which contains the indices of the leaders. Particularly, all the quadrotors are leaders, when $\mathcal{V}_{L}=\mathcal{V}$. The indices of the followers are contained in the complementary set of $\mathcal{V}_{L}$, namely, $\mathcal{V}-\mathcal{V}_{L}$.

The interaction matrix $G$ for L-F formation is defined as follows

$$
G=G^{D}-G^{A}+G^{L}
$$

Let us note that the part $G^{D}-G^{A}$ is normally called the Laplacian in graph theory. Since we are concerned by the L-F formation here, we use the interaction matrix $G$ to represent the interactions of agents. The interaction matrix $G$ is symmetric. Obviously, if no leader exists in the group, namely, leaderless formation structure, the matrix $G^{L}$ will be equal to zero. In this case, the matrix $G$ is equal to the Laplacian of the graph.

\section{B. Decentralized consensus algorithm}

According to Olfati [26], a consensus algorithm (or protocol) is an interaction rule that specifies the information exchange between an agent and all of its neighbors on the network. Specifically, if the neighbors of an agent are local, then, the consensus algorithm is decentralized. On the contrary, if each agent has information exchange with all the other agents, the consensus algorithm is centralized. Furthermore, 
any global information should not be used by all the agents, otherwise, the consensus algorithm is also centralized.

Let us denote the "tracking error" by

$$
e_{i}=x_{i}-r-d_{i 0}
$$

between agent $i$ and a sufficient smooth reference trajectory $r(t)$ of the formation. The scalar $d_{i 0}$ represents the desired distance between agent $i$ and the reference trajectory. Different assignments of the scalars $d_{i 0}$ lead different formation shapes. The scalars $d_{i 0}$ can be constant or variable. In this paper, without loss of generality, we consider a formation with constant shape, i.e. $d_{i 0}, i \in \mathcal{V}$ are constants. We call this kind of formation "rigid formation". The objective of the neighborbased consensus algorithm for the leader-follower formation of multiple agents is to guarantee that the errors $e_{i}, i \in \mathcal{V}$ converge to zero.

\section{Properties of interaction matrix}

In this subsection, we introduce two important properties of the interaction matrix through two propositions. These properties will be used in the following sections.

Proposition 1. Let $\mathcal{G}$ be an undirected simple graph, then the interaction matrix $G$ in equation (1), is positive-definite, if i) $\mathcal{G}$ is connected; ii) the multi-agent system has at least one leader.

Proof. Since $\mathcal{G}$ is connected, we have $G^{D}-G^{A} \geq 0$. Considering the definition of $G^{L}$, we have $G^{L} \geq 0$. We prove this proposition by contradiction. Firstly, we suppose that there exists a nonzero vector $x \in \mathbb{R}^{n}$, which renders

$$
x^{T} G x=x^{T}\left(L+G^{L}\right) x=x^{T} L x+x^{T} G^{L} x=0
$$

Therefore, we must have $x^{T} L x=0$ and $x^{T} G^{L} x=0$.

Since $x^{T} L x=0$, we obtain that $x=\alpha \mathbf{1}_{n}$, where $\alpha$ is a nonzero scalar. According to the fact that a leader exists, then, $G^{L} \neq 0$. As a result, $x^{T} G^{L} x=\alpha^{2} \mathbf{1}_{n}^{T} G^{L} \mathbf{1}_{n}>0$, which contradicts $x^{T} G^{L} x=0$. Therefore, such a nonzero vector $x$ does not exist. Thus, for any nonzero vector $x, x^{T} G x>0$, namely, $G$ is positive-definite.

When the condition $i$ ) is not satisfied such that $\mathcal{G}$ is not connected, the graph can be divided into several connected sub-graphs. In that case, according to the proposition 11, we have the following corollary.

Corollary 1. The interaction matrix $G$ is positive-definite, if each sub-group of the agents, which is described by a connected sub-graph, has at least one leader.

Proof. If a multi-agent system contains several connected subgroups of agents, then, the interaction matrix is block diagonal. Since each sub-group is connected and has at least one leader, then, the block in the interaction matrix is positive definite. Hence, the interaction matrix is positive definite.

Proposition 2. The minimum eigenvalue of the interaction matrix $G$ for an undirected connected graph $\mathcal{G}$ will increase, if extra edges are added.
Proof. The graph can be represented by $\mathcal{G}=(\mathcal{V}, \mathcal{E})$. The corresponding interaction matrix is $G$. We construct another graph $\mathcal{G}_{e}=\left(\mathcal{V}, \mathcal{E}_{e}\right)$, where the vertices set is the same as the graph $\mathcal{G}$, while the edge set is composed by extra added edges. Its interaction matrix is denoted by $G_{e}$. Then, the graph $\mathcal{G}$ becomes $\mathcal{G}^{\prime}=\mathcal{G} \cup \mathcal{G}_{e}$ after extra edges have been added. The interaction matrix for graph $\mathcal{G}^{\prime}$ is denoted by $G^{\prime}$, then, we have

$$
G^{\prime}=G+G_{e}
$$

According to Courant-Fischer theorem [27], $\lambda_{\min }\left(G^{\prime}\right)=\min _{\|x\|=1} x^{T} G^{\prime} x=\min _{\|x\|=1} x^{T}\left(G+G_{e}\right) x>$ $\min _{\|x\|=1} x^{T} G x+\min _{\|x\|=1} x^{T} G_{e} x=\lambda_{\min }(G)+$ $\lambda_{\min }\left(G_{e}\right) \geq \lambda_{\min }(G)$. Then, the result is obtained.

Corollary 2. The minimum eigenvalue of the interaction matrix for an undirected connected graph $\mathcal{G}$ will increase, if more agent(s) are assigned as leader(s).

Proof. The proof is similar to the proof of proposition 2 .

The normalized interaction matrix is defined by $\bar{G}=$ $\left(G^{D}+G^{L}\right)^{-1} G$. The normalized interaction matrix $\bar{G}$ is not symmetric, but it has the following property.

Proposition 3. The normalized interaction matrix $\bar{G}$ has real eigenvalues, if $\mathcal{G}$ is undirected.

Proof. Since $\bar{G}$ is a normalized interaction matrix, then, $\bar{G}=$ $\left(G^{D}+G^{L}\right)^{-1} \cdot G$. We recall that $G$ is symmetric.

Using $\left(G^{D}+G^{L}\right)^{-\frac{1}{2}}$, we apply similarity transformation to $\bar{G}$, then, we obtain that $\left(G^{D}+G^{L}\right)^{\frac{1}{2}} \cdot \bar{G} \cdot\left(G^{D}+G^{L}\right)^{-\frac{1}{2}}=$ $\left(G^{D}+G^{L}\right)^{-\frac{1}{2}} \cdot G \cdot\left(G^{D}+G^{L}\right)^{-\frac{1}{2}}$ is symmetric, because $G^{D}+G^{L}$ are diagonal and $G$ is symmetric.

Since $\bar{G}$ is similar to $\left(G^{D}+G^{L}\right)^{-\frac{1}{2}} \cdot G \cdot\left(G^{D}+G^{L}\right)^{-\frac{1}{2}}$, the eigenvalues of $\bar{G}$ are real, moreover, they are equal to the eigenvalues of $\left(G^{D}+G^{L}\right)^{-\frac{1}{2}} \cdot G \cdot\left(G^{D}+G^{L}\right)^{-\frac{1}{2}}$.

\section{CONTROLLER DESIGN}

In this section, the formation of a group of quadrotors with L-F configuration is considered. Among the UAVs, one or several UAVs are leader, while other UAVs are followers. The objective of the formation is to track a given reference formation trajectory (RFT), i.e. guidance of multiple quadrotors. We firstly show that such a formation trajectory tracking problem is an L-F consensus problem. Then, considering actuator saturation and formation performance, the basic consensus algorithm is developed and implemented on the formation of quadrotors.

\section{A. L-F consensus of quadrotors}

The rigid formation problem is investigated in this paper. A formation is rigid if the desired inter-distance between each pair of UAVs does not change over time. The UAVs keep a certain pattern and track a RFT. This type of formation has applications such as object transportation [4] and surveillance of area of interest [15].

We consider the planar formation of UAVs, since the altitude control is decoupled with the planar motion control. We denote by $x_{i}=\left[X_{i}, Y_{i}\right]^{T}$ and $\dot{x}_{i}=\left[\dot{X}_{i}, \dot{Y}_{i}\right]^{T}$ the planar position 
and velocity vectors for UAV $i$. The formation controller of each UAV uses the relative positions and velocities with respect to its neighbors, which can be represented by vectors $\left[\left(x_{i}-x_{j}\right)^{T},\left(\dot{x}_{i}-\dot{x}_{j}\right)^{T}\right]^{T}, j \in \mathcal{N}_{i}$. If the UAV is a leader, besides the foregoing measurements, its formation controller also uses the relative position and velocity with respect to the reference trajectory $\left[\left(x_{i}-r(t)\right)^{T},\left(\dot{x}_{i}-\dot{r}(t)\right)^{T}\right]^{T}$, where we denote by $r(t)=\left[r_{X}(t), r_{Y}(t)\right]^{T}$ the reference formation trajectory (RFT).

The objective of the rigid formation is to guarantee that all the quadrotors track the RFT with some constant biases $d_{i 0}=\left[d_{i 0}^{X}, d_{i 0}^{Y}\right] \in \mathbb{R}^{2}$, such that the quadrotors keep a constant formation pattern.

We are now ready to state a (slightly modified) version of the L-F consensus scheme proposed in [28].

Definition 1. The L-F consensus of multiple quadrotors is said to be achieved if, for each $U A V i \in \mathcal{V}$,

$$
\begin{aligned}
& \lim _{t \rightarrow \infty}\left\|x_{i}-r(t)-d_{i 0}\right\|=0 \\
& \lim _{t \rightarrow \infty}\left\|\dot{x}_{i}-\dot{r}(t)\right\|=0
\end{aligned} \quad \text { where } i=1, \ldots, n
$$

for some initial condition $x_{i}(0), i=1, \ldots, n$.

Therefore, the desired position of UAV $i \in \mathcal{V}$ evolves according to $x_{i}^{d}(t)-r(t)=d_{i 0}$ and $\dot{x}_{i}^{d}(t)-\dot{r}(t)=0$, then, we obtain

$$
x_{i}^{d}(t)=d_{i 0}+r(t) \text { and } \dot{x}_{i}^{d}(t)=\dot{r}(t)
$$

Then, the rigid formation task contains two parts, i) the desired trajectory RFT $r(t)$; ii) the biases away from the RFT. An example of four UAVs with rigid formation task is shown in Fig 1(a), where the solid red circle represents the RFT at time $t_{a}$. The dashed red circles represent the desired positions $x_{i}^{d}\left(t_{a}\right)$ for the UAVs $i \in \mathcal{V}$ at $t_{a}$. The solid black circles represent the quadrotors real positions at $t_{a}$, i.e. $x_{i}\left(t_{a}\right), i \in \mathcal{V}$.

However, the RFT $r(t)$ is not available for the followers, therefore the desired trajectory $x_{i}^{d}(t)$ for UAV $i$ is not available, in other words, $x_{i}^{d}(t)$ cannot be used in the formation controller design for the followers. For the followers, only the neighbors states are available for the formation controller design, as shown in Fig 1 (b). Then, the formation problem becomes: how to find the available trajectories for UAVs $i \in \mathcal{V}$ in order to attain the formation task.

Let us firstly make a sum of the relative position state vectors. Note that we drop the explicit expression of time in the expressions for the sake of simplicity.

$$
\begin{array}{cc}
\sum_{j \in \mathcal{N}_{i}}\left(x_{i}-x_{j}-d_{i j}\right) & \text { if } i \in \mathcal{V}-\mathcal{V}_{L} \\
\sum_{j \in \mathcal{N}_{i}}\left(x_{i}-x_{j}-d_{i j}\right)+x_{i}-r-d_{i 0} & \text { if } i \in \mathcal{V}_{L}
\end{array}
$$

The desired inter-distance of UAV $i$ and $j$ is given by $d_{i j}=$ $d_{i 0}-d_{j 0}$. Then, equations (4) can be rewritten as follows

$\sum_{j \in \mathcal{N}_{i}}\left(x_{i}-r-d_{i 0}-\left(x_{j}-r-d_{j 0}\right)\right)$ if $i \in \mathcal{V}-\mathcal{V}_{L}$
$\sum_{j \in \mathcal{N}_{i}}\left(x_{i}-r-d_{i 0}-\left(x_{j}-r-d_{j 0}\right)\right)+x_{i}-r-d_{i 0}$ if $i \in \mathcal{V}_{L}$

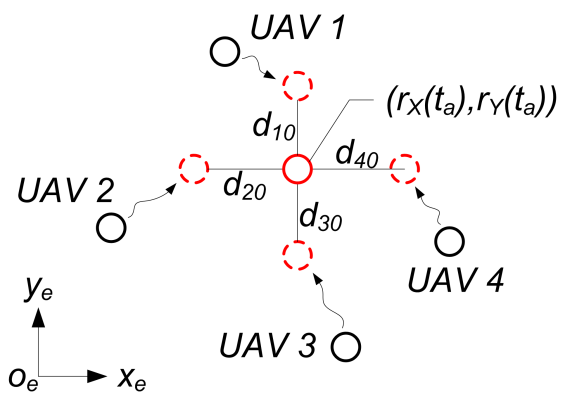

(a) Rigid formation task

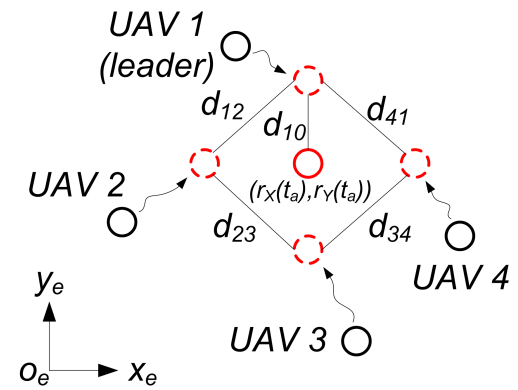

(b) Available trajectory for each UAV

Fig. 1: Rigid formation task for four UAVs, where UAV 1 is a leader. The objective is to track the trajectory $r(t)$ with constant biases $d_{10}=[0, \sqrt{2}]^{T}, d_{20}=[-\sqrt{2}, 0]^{T}, d_{30}=[0,-\sqrt{2}]^{T}$ and $d_{40}=[\sqrt{2}, 0]^{T}$.

Let us denote "formation error" by

$\mathrm{e}_{i}=\left\{\begin{array}{c}x_{i}-\frac{1}{\left|\mathcal{N}_{i}\right|} \sum_{j \in \mathcal{N}_{i}}\left(x_{j}+d_{i j}\right) \quad \text { if } i \in \mathcal{V}-\mathcal{V}_{L} \\ x_{i}-\frac{1}{\left|\mathcal{N}_{i}+1\right|}\left(\sum_{j \in \mathcal{N}_{i}}\left(x_{j}+d_{i j}\right)+r+d_{i 0}\right) \quad \text { if } i \in \mathcal{V}_{L}\end{array}\right.$

We rewrite the equation (4) in matrix form for all the quadrotors as follows

$$
\left[\begin{array}{c}
\mathrm{e}_{1} \\
\vdots \\
\mathrm{e}_{n}
\end{array}\right]=\left(\bar{G} \otimes I_{2}\right)\left[\begin{array}{c}
e_{1} \\
\vdots \\
e_{n}
\end{array}\right]
$$

where $\bar{G}$ represents the normalized interaction matrix. According to proposition 1, corollary 1, we know that $\lambda_{\min }(\bar{G})>0$ if the graph of the multi-UAV system is connected with at least one leader or each connected subgraph has at least one leader. We suppose these conditions are satisfied. Thus, if the formation error $e_{i}$ for each UAV converges to zero, the formation task is achieved. Note that $d_{i j}$ is constant in a rigid formation task. The goal is to design a formation controller, which guarantees the convergence of $\mathrm{e}_{i}$.

\section{B. Attitude and altitude control}

The dynamics of a quadrotor can be generally divided into two parts, namely, the rotational dynamics in inner-loop, and the translational dynamics in outer-loop respectively. The inputs of the translational dynamics are the attitude angles and the total thrust force, while the outputs of the translational 
dynamics are the positions and velocities of the quadrotor in the inertial frame.

According to [29], the attitude controller is given in the following process. We firstly define two matrices as follows

$$
T_{i}=\left[\begin{array}{ccc}
1 & 0 & -\sin \theta_{i} \\
0 & \cos \phi_{i} & \cos \theta_{i} \sin \phi_{i} \\
0 & -\sin \phi_{i} & \cos \theta_{i} \cos \phi_{i}
\end{array}\right]
$$

and

$$
\tilde{T}_{i}=\left[\begin{array}{ccc}
\tan \theta_{i} & 0 & \cos \theta_{i}+\tan \theta_{i} \sin \theta_{i} \\
0 & -\cos \theta_{i} & 0 \\
\sec \theta_{i} & 0 & \tan \theta_{i}
\end{array}\right],
$$

We define a map $S(\cdot): \mathbb{R}^{3} \rightarrow \mathbb{R}^{3 \times 3}$, which satisfies the property $\boldsymbol{S}\left(v_{1}\right) \cdot v_{2}=v_{1} \times v_{2}$, for two given arbitrary vectors $v_{1}, v_{2} \in \mathbb{R}^{3}$. Then, the torque vector $\tau_{i}$ is designed as follows

$$
\tau_{i}=J T_{i} \bar{\tau}_{i}-J T_{i} \tilde{T}_{i}\left[\begin{array}{c}
\dot{\phi}_{i} \dot{\theta}_{i} \\
\dot{\phi}_{i} \dot{\psi}_{i} \\
\dot{\theta}_{i} \dot{\psi}_{i}
\end{array}\right]-S^{T}\left(T_{i} \cdot \dot{\Theta}_{i}\right) J T_{i} \cdot \dot{\Theta}_{i}
$$

where

$$
\bar{\tau}_{i}=\ddot{\Theta}_{i}^{d}-\frac{k_{2 \Theta_{i}}}{\epsilon}\left(\dot{\Theta}_{i}-\dot{\Theta}_{i}^{d}\right)-\frac{k_{1 \Theta_{i}}}{\epsilon^{2}}\left(\Theta_{i}-\Theta_{i}^{d}\right)
$$

In (8), $k_{1 \Theta_{i}}$ and $k_{2 \Theta_{i}}$ represent two diagonal positive-definite gain matrices. Notations $\Theta_{i}^{d}, \dot{\Theta}_{i}^{d}$ and $\ddot{\Theta}_{i}{ }^{d}$ represent the desired Euler angles vector and their derivatives. The scalar $\epsilon \in(0,1]$.

According to [29], we have two remarks on the use of the attitude controller (7).

a) Remark 1: If the scalar " $\epsilon$ " in (8) is selected sufficiently small, the dynamics of a quadrotor has a two time-scale property. The model of the quadrotor can be decomposed into the slow (reduced) model, i.e. translational dynamics and fast (boundary-layer) model, i.e. rotational dynamics. We use the following example to illustrate this fact.

Example 1. The trajectory tracking of a single quadrotor is studied. The objective is to track a circular trajectory. The tracking curves of the quadrotor with different $\epsilon$ are given in Fig.2.

We can observe from Fig 2 that the real outputs of the complete model approach the outputs of the reduced model faster, if $\epsilon$ is selected smaller. Thus, by selecting some proper " $\epsilon$ ", we can use the reduced model instead of the complete model in the study of the formation controller design, which will greatly simplify the problem.

b) Remark 2: The dynamics of pitch, roll and yaw are decoupled.

In this work, the desired altitude $Z_{i}^{d}$ of each UAV is constant. In other words, the formation takes place in $o_{e} x_{e} y_{e}$ plan. The thrust force is given as follows

$$
F_{T_{i}}=\frac{m g+m \cdot u_{i}^{Z}}{\cos \theta_{i} \cos \phi_{i}}
$$

where $u_{i}^{Z}$ represents the altitude controller.

By using (9), the altitude control is decoupled with the planar translational dynamics $\left(X_{i}\right.$ and $\left.Y_{i}\right)$. Thus, we give the altitude controller as follows

$$
u_{i}^{Z}=\sigma_{b}\left(-k_{2 Z} \dot{Z}_{i}-k_{1 Z}\left(Z_{i}-Z_{i}^{d}\right)\right)
$$
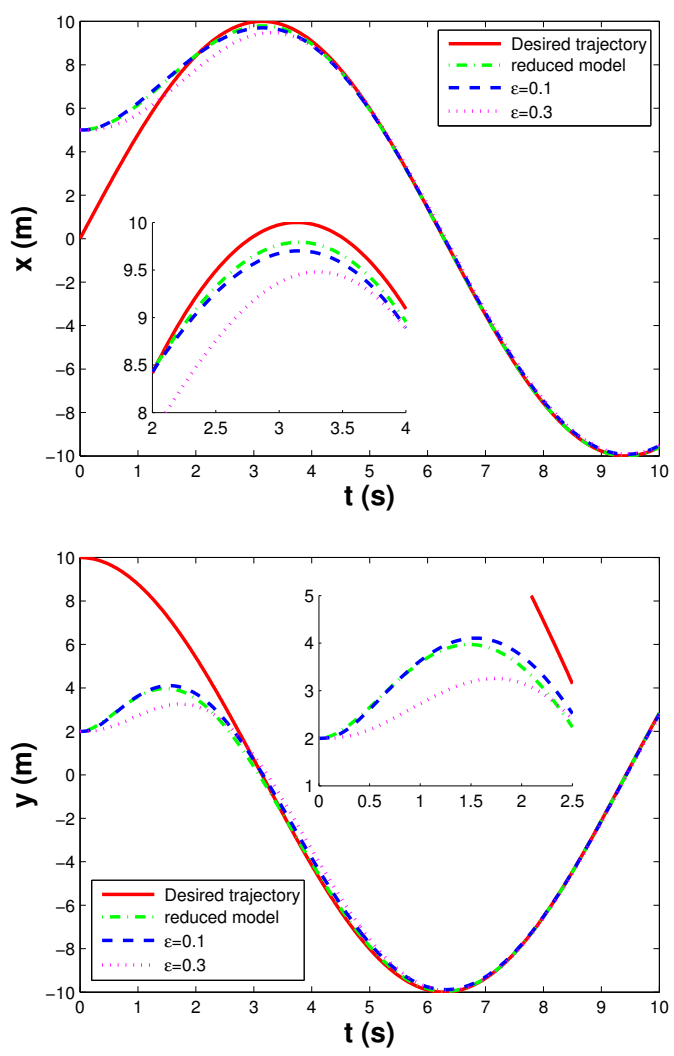

Fig. 2: Navigation control based on singular perturbed theory

Function $\sigma_{b}(\cdot)$ represents a standard saturation function.

$$
\sigma_{b}(a)=\operatorname{sgn}(a) \min \{b,|a|\}
$$

where "sgn" represents the sign function. We select $b=1$. The controller (10) asymptotically stabilizes the altitude.

Since the altitude control is decoupled with the planar translational dynamics, the convergence of $Z_{i}$ do not depend on the other states of the quadrotors except $Z_{i}$ and $\dot{Z}_{i}$. When the altitude of the quadrotors gets stabilized, $u_{i}^{Z} \approx 0$.

Now, we give the formation controller design in the following part of this section.

Since the planar translational motions are generated by the attitude angles, the formation controller is to obtain the proper desired attitude angles $\Theta_{i}^{d}$. If we design the desired attitude angles as follows

$$
\begin{aligned}
\theta_{i}^{d} & =\arctan \left(\frac{u_{i}^{X}}{g}\right) \\
\phi_{i}^{d} & =\arctan \left(\frac{-u_{i}^{Y} \cos \left(\arctan \left(u_{i}^{X} / g\right)\right)}{g}\right) \\
\psi_{i}^{d} & =0
\end{aligned}
$$

where $u_{i}^{X}$ and $u_{i}^{Y}$ represent the formation controllers in direction $x_{e}$ and $y_{e}$ in the inertial frame. Then, the planar translational dynamics of UAV $i$ yields

$$
\begin{aligned}
\ddot{X}_{i} & =u_{i}^{X}+\delta_{i}^{X} \\
\ddot{Y}_{i} & =u_{i}^{Y}+\delta_{i}^{Y}
\end{aligned}
$$


The terms $\delta_{i}^{X}$ and $\delta_{i}^{Y}$ have the following expression.

$$
\begin{aligned}
\delta_{i}^{X}=\left(g+u_{i}^{Z}\right) \cdot & \left(\sin \Delta \psi_{i} \cdot \frac{\tan \left(\phi_{i}^{d}+\Delta \phi_{i}\right)}{\cos \left(\theta_{i}^{d}+\Delta \theta_{i}\right)}+\right. \\
& \left.\cos \Delta \psi_{i} \cdot \tan \left(\theta_{i}^{d}+\Delta \theta_{i}\right)-\frac{g}{g+u_{i}^{Z}} \tan \theta_{i}^{d}\right) \\
\delta_{i}^{Y}=\left(g+u_{i}^{Z}\right) \cdot & \left(-\cos \Delta \psi_{i} \cdot \frac{\tan \left(\phi_{i}^{d}+\Delta \phi_{i}\right)}{\cos \left(\theta_{i}^{d}+\Delta \theta_{i}\right)}+\right. \\
& \left.\sin \Delta \psi_{i} \cdot \tan \left(\theta_{i}^{d}+\Delta \theta_{i}\right)+\frac{g}{g+u_{i}^{Z}} \frac{\tan \phi_{i}^{d}}{\cos \theta_{i}^{d}}\right)
\end{aligned}
$$

where $\Delta \phi_{i}, \Delta \theta_{i}$ and $\Delta \psi_{i}$ represent the tracking error of the attitude angles $\Theta_{i}-\Theta_{i}^{d}=\left[\Delta \phi_{i}, \Delta \theta_{i}, \Delta \psi_{i}\right]$. We can observe that $\delta_{i}^{X}$ and $\delta_{i}^{Y}$ are in terms of $u_{i}^{Z}$, and $\Theta_{i}-\Theta_{i}^{d}$. According to [29], the attitude tracking error $\Theta_{i}-\Theta_{i}^{d}$ is bounded, if $\Theta_{i}^{d}$ is bounded. Furthermore, $\Theta_{i}-\Theta_{i}^{d}$ exponentially converges to the origin. Additionally, $\left|u_{i}^{Z}\right| \leq 1$ and $u_{i}^{Z} \rightarrow 0$ when the altitude is stabilized. Thus, we obtain that $\delta_{i}^{X}$ and $\delta_{i}^{Y}$ are bounded.

\section{Formation control}

We firstly consider the nominal model of (13) by setting $\delta_{i}^{X}$ and $\delta_{i}^{Y}$ null. The dynamics of $X_{i}$ and $Y_{i}$ are similar, thus, we consider the dynamics of $X_{i}$ for example. Note that in the sequel, the superscript " $X$ " is omitted for the sake of simplicity. Then, we have

$$
\ddot{X}_{i}=u_{i}
$$

Let us denote by $e_{i X}=X_{i}-\bar{X}_{i}^{d}$ the formation error in $x_{e}$ direction. Let us define a nonlinear function $\rho_{i}: \mathbb{R}^{2} \rightarrow \mathbb{R}$. We propose the formation controller as follows

$$
\begin{gathered}
u_{i}=-M \tanh \left(k_{2} \dot{e}_{i X}+k_{1} e_{i X}\right) \\
-M \tanh \left(\rho_{i}\left(e_{i X}, \dot{e}_{i X}\right)\right)+\ddot{\bar{X}}_{i}^{d} \\
\rho_{i}\left(e_{i X}, \dot{e}_{i X}\right)=k_{N} \dot{e}_{i X}
\end{gathered}
$$

where $M$ represents a positive constant scalar. $k_{1}$ and $k_{2}$ are two positive constant gains. The symbol $k_{N}$ represents a positive nonlinear gain. For example, we can select $k_{N}$ as follows

$$
k_{N}=\eta_{1} \exp ^{-\eta_{2} e_{i X}^{2}}
$$

where $\eta_{1} \geq 0, \eta_{2}>0$. The selection of $k_{N}$ in 16 has a physical meaning. When the formation error $e_{i X}$ is large, the effect of $\rho_{i}\left(e_{i X}, \dot{e}_{i X}\right)$ is small. In contrary, when $e_{i X}$ approaches zero, the nonlinear gain $k_{N}$ increases, which can reduce the approaching velocity, such that the overshoot is small.

Proposition 4. The formation controller $u_{i}$ (15) is bounded.

Proof. According to [5, the desired trajectory $\bar{X}_{i}^{d}$ for UAV $i$ satisfies

$$
\bar{X}_{i}^{d}=\left\{\begin{array}{cc}
\frac{1}{\left|\mathcal{N}_{i}\right|} \sum_{j \in \mathcal{N}_{i}}\left(X_{j}+d_{i j}\right) & \text { if } i \in \mathcal{V}-\mathcal{V}_{L} \\
\frac{1}{\left|\mathcal{N}_{i}+1\right|}\left(\sum_{j \in \mathcal{N}_{i}}\left(X_{j}+d_{i j}\right)+r_{X}+d_{i 0}\right) & \text { if } i \in \mathcal{V}_{L}
\end{array}\right.
$$

where $d_{i j}, d_{i 0} \in \mathbb{R}$ (recall that the subscript " $X$ " has been omitted here) represent some constant offset scalar. Then,

$$
\dot{\bar{X}}_{i}^{d}=\left\{\begin{array}{cc}
\frac{1}{\left|\mathcal{N}_{i}\right|} \sum_{j \in \mathcal{N}_{i}} \dot{X}_{j} & \text { if } i \in \mathcal{V}-\mathcal{V}_{L} \\
\frac{1}{\left|\mathcal{N}_{i}+1\right|}\left(\sum_{j \in \mathcal{N}_{i}} \dot{X}_{j}+\dot{r}_{X}\right) & \text { if } i \in \mathcal{V}_{L}
\end{array}\right.
$$

Let us denote by $\bar{u}_{i}=k_{2} \dot{e}_{i X}+k_{1} e_{i X}$. Then, if $i \in \mathcal{V}-\mathcal{V}_{L}$,

$$
\ddot{\bar{X}}_{i}^{d}=\frac{1}{\left|\mathcal{N}_{i}\right|} \sum_{j \in \mathcal{N}_{i}}\left(-M \tanh \bar{u}_{j}-M \tanh \rho_{j}+\ddot{\bar{X}}_{j}^{d}\right)
$$

such that

$\ddot{\bar{X}}_{i}^{d}-\frac{1}{\left|\mathcal{N}_{i}\right|} \sum_{j \in \mathcal{N}_{i}} \ddot{X}_{j}^{d}=-\frac{1}{\left|\mathcal{N}_{i}\right|} \sum_{j \in \mathcal{N}_{i}}\left(M \tanh \bar{u}_{j}+M \tanh \rho_{j}\right)$

if $i \in \mathcal{V}_{L}$,

$\ddot{\bar{X}}_{i}^{d}=\frac{1}{\left|\mathcal{N}_{i}+1\right|}\left(\sum_{j \in \mathcal{N}_{i}}\left(-M \tanh \bar{u}_{j}-M \tanh \rho_{j}+\ddot{\bar{X}}_{j}^{d}\right)+\ddot{r}_{X}\right)$

such that

$$
\begin{aligned}
\ddot{\bar{X}}_{i}^{d} & -\frac{1}{\left|\mathcal{N}_{i}+1\right|} \sum_{j \in \mathcal{N}_{i}} \ddot{X}_{j}^{d} \\
& =-\frac{1}{\left|\mathcal{N}_{i}+1\right|}\left(\sum_{j \in \mathcal{N}_{i}}\left(M \tanh \bar{u}_{j}+M \tanh \rho_{j}\right)-\ddot{r}_{X}\right)
\end{aligned}
$$

Without loss of generality, we assume that UAVs $1 \sim i$ are leaders, while $i+1 \sim n$ are followers. Then, we rewrite the foregoing equations for all the quadrotors in matrix form as follows

$$
\left[\begin{array}{c}
\ddot{\bar{X}}_{1}^{d} \\
\vdots \\
\ddot{\bar{X}}_{n}^{d}
\end{array}\right]=G^{-1} \cdot\left[\begin{array}{c}
-\frac{1}{\left|\mathcal{N}_{1}+1\right|}\left(\sum_{j \in \mathcal{N}_{1}}\left(M \tanh \bar{u}_{j}+M \tanh \rho_{j}\right)-\ddot{r}_{X}\right) \\
\vdots \\
-\frac{1}{\left|\mathcal{N}_{i}+1\right|}\left(\sum_{j \in \mathcal{N}_{i}}\left(M \tanh \bar{u}_{j}+M \tanh \rho_{j}\right)-\ddot{r}_{X}\right) \\
-\frac{1}{\left|\mathcal{N}_{i+1}\right|} \sum_{j \in \mathcal{N}_{i+1}}\left(M \tanh \bar{u}_{j}+M \tanh \rho_{j}\right) \\
\vdots \\
-\frac{1}{\left|\mathcal{N}_{n}\right|} \sum_{j \in \mathcal{N}_{n}}\left(M \tanh \bar{u}_{j}+M \tanh \rho_{j}\right)
\end{array}\right]
$$

The RFT is usually selected such that its derivatives are bounded. Then, we observe that $\ddot{\bar{X}}_{i}^{d}, i \in \mathcal{V}$ are bounded, since the function $\tanh (\cdot)$ is bounded by 1 . Therefore, we conclude that $u_{i}, i \in \mathcal{V}$ are bounded.

Substituting (15) into the first equation in (14), we obtain

$$
\ddot{e}_{i X}=-M \tanh \bar{u}_{i}-M \tanh \rho_{i}
$$

Proposition 5. The origin of (18) is globally asymptotically stable, if i) $k_{N}$ is given by (16); ii) the gains in $\bar{u}_{i}$ satisfy $k_{1} \geq \eta_{1} k_{2} M$.

Proof. Let us define a continuously differentiable, radially unbounded positive-definite function as follows

$$
V_{i}=\ln \cosh \left(k_{2} \dot{e}_{i X}+k_{1} e_{i X}\right)+\frac{k_{1}}{2 M} \dot{e}_{i X}^{2}
$$


$V_{i}=0$, if and only if $e_{i X}=0$ and $\dot{e}_{i X}=0$.

Then, the derivative of $V_{i}$ yields

$$
\begin{aligned}
\dot{V}_{i}= & \tanh \bar{u}_{i} \cdot \dot{\bar{u}}_{i}+\frac{k_{1}}{M} \dot{e}_{i X} \ddot{e}_{i X} \\
= & \tanh \bar{u}_{i}\left(k_{2} \ddot{e}_{i X}+k_{1} \dot{e}_{i X}\right) \\
& +\frac{k_{1}}{M} \dot{e}_{i X}\left(-M \tanh \bar{u}_{i}-M \tanh \rho_{i}\right) \\
= & \tanh \bar{u}_{i}\left(k_{2}\left(-M \tanh \bar{u}_{i}-M \tanh \rho_{i}\right)+k_{1} \dot{e}_{i X}\right) \\
& -k_{1} \dot{e}_{i X}\left(\tanh \bar{u}_{i}+\tanh \rho_{i}\right) \\
= & -k_{2} M \tanh ^{2} \bar{u}_{i}-k_{2} M \tanh \bar{u}_{i} \tanh \rho_{i} \\
& -k_{1} \dot{e}_{i X} \tanh \rho_{i}
\end{aligned}
$$

Then, we have

$$
\begin{aligned}
\dot{V}_{i} \leq-k_{2} M \tanh ^{2}\left(\bar{u}_{i}\right) & -k_{1} \dot{e}_{i X} \tanh \rho_{i} \\
& +k_{2} M\left|\tanh \bar{u}_{i}\right|\left|\tanh \rho_{i}\right|
\end{aligned}
$$

According to $(15), \rho_{i}$ has the same sign as $\dot{e}_{i X}$. Then, $-k_{1} \dot{e}_{i X} \tanh \rho_{i} \leq 0$.

- Case 1: If $\left|\rho_{i}\right| \leq\left|\bar{u}_{i}\right|,\left|\tanh \rho_{i}\right| \leq\left|\tanh \bar{u}_{i}\right|$. Then, $\left|\tanh \rho_{i}\right|\left|\tanh \bar{u}_{i}\right| \leq \tanh ^{2} \bar{u}_{i}$. Therefore,

$$
\dot{V}_{i} \leq-k_{1} \dot{e}_{i X} \tanh \rho_{i} \leq 0
$$

We obtain that $\dot{V}_{i}$ is semi-definite negative. We then invoke here the LaSalle's invariance principle. Let us compute the largest invariant set where $\dot{V}_{i}=0$, such that equation (20) equal to zero. We consider the following cases.

- Case a: if $\dot{e}_{i X} \neq 0$, then, according to $21, \dot{V}_{i}<0$, which contradicts $\dot{V}_{i}=0$.

- Case b: if $\dot{e}_{i X}=0$, then, according to 20, $\dot{V}_{i}=$ $-k_{2} M \tanh ^{2}\left(k_{1} e_{i X}\right)=0$, which implies $e_{i X}=0$.

Therefore, in Case 1, the largest invariant set contains only the origin.

- Case 2: If $\left|\rho_{i}\right|>\left|\bar{u}_{i}\right|,\left|\rho_{i}\right|>\left|\tanh \bar{u}_{i}\right| \geq 0$. Since $k_{1} \geq$ $\eta_{1} k_{2} M$, then, we have $k_{2} M\left|\tanh \bar{u}_{i}\right|<k_{1}\left|\dot{e}_{i X}\right|$. Thus,

$$
\dot{V}_{i} \leq-k_{2} M \tanh ^{2} \bar{u}_{i} \leq 0
$$

We obtain that $\dot{V}_{i}$ is semi-definite negative. As mentioned before, we compute the largest invariant set where $\dot{V}_{i}=0$, such that equation 20 equal to zero. We consider the following cases.

- Case a: if $\left|\bar{u}_{i}\right| \neq 0$, then, according to $22, \dot{V}_{i}<0$, which contradicts $\dot{V}_{i}=0$.

- Case b: if $\left|\bar{u}_{i}\right|=0$, then, according to 20, $\dot{V}_{i}=$ $-k_{1} \dot{e}_{i X} \tanh \rho_{i}=0$, which implies $\dot{e}_{i X}=0$. Since $\bar{u}_{i}=0$, we obtain that $e_{i X}=0$.

Therefore, in Case 2, the largest invariant set contains only the origin.

Since the largest invariant set contains only the origin, then, according to the LaSalle theorem, the origin of the system (18) is globally asymptotically stable.

Remark 1. The decoupled property of $X$ and $Y$ dynamics permits us to design the formation controller separately, the design procedure of $u_{i}^{Y}$ is the same as $u_{i}^{X}$, such that

$u_{i}^{Y}=-M \tanh \left(k_{2} \dot{e}_{i Y}+k_{1} e_{i Y}\right)-M \tanh \rho_{i}\left(e_{i Y}, \dot{e}_{i Y}\right)+\ddot{\bar{Y}}_{i}^{d}$
Remark 2. The desired attitude angles for each UAV are obtained by substituting (15) and (23) into (12). Owing to the use of the hyperbolic tangent function, $u_{i}^{X}$ and $u_{i}^{Y}$ are high-order differentiable, such that the derivatives $\dot{\theta}_{i}^{d}, \ddot{\theta}_{i}^{d}$ and $\dot{\phi}_{i}^{d}, \ddot{\phi}_{i}^{d}$ are bounded.

Let us reconsider the terms $\delta_{i}^{X}$ and $\delta_{i}^{Y}$ in 13 caused by the tracking error of the attitude angles. We introduce our main result in the following theorem.

Theorem 1. Let $G$ be the interaction matrix of an $L-F$ formation of quadrotors with constant topology. The formation controllers are given in (15) and (23). The attitude is controlled by (7). The altitude is controlled by (9) where $u_{i}^{Z}$ is given in (10). Then, $e_{i X}$ and $e_{i Y}$ converge to zero asymptotically, if i) $G$ is invertible, ii) the initial velocity of each $U A V$ is finite.

Proof. The terms $\delta_{i X}$ and $\delta_{i Y}$ are in terms of the tracking errors of the attitude angles $\left(\Delta \phi_{i}, \Delta \theta_{i}\right.$ and $\left.\Delta \psi_{i}\right)$ and $u_{i}^{Z}$. According to $177, \sqrt{15}, \sqrt{23}$ and $\left(10\right.$, , the controllers $u_{i}^{X}, u_{i}^{Y}$ and $u_{i}^{Z}$ are bounded. Then, according to $(12)$, we know that $\theta_{i}^{d}, \phi_{i}^{d}$ and $\psi_{i}^{d}$ are bounded. The attitude angles are controlled by (7), then, $\Delta \phi_{i}, \Delta \theta_{i}$ and $\Delta \psi_{i}$ are bounded. Therefore, $\delta_{i X}$ and $\delta_{i Y}$ are bounded.

We take $e_{i X}$ for example. Then, (18) becomes

$$
\ddot{e}_{i X}=-M \tanh \bar{u}_{i}-M \tanh \rho_{i}+\delta_{i X}
$$

The derivative of the positive semidefinite function $V_{i}$ in 19 p yields

$$
\begin{aligned}
\dot{V}_{i}= & \tanh \bar{u}_{i} \cdot \dot{\bar{u}}_{i}+\frac{k_{1}}{M} \dot{e}_{i X} \ddot{e}_{i X} \\
= & \tanh \bar{u}_{i}\left(k_{2} \ddot{e}_{i X}+k_{1} \dot{e}_{i X}\right) \\
& -\frac{k_{1}}{M} \dot{e}_{i X}\left(M \tanh \bar{u}_{i}+M \tanh \rho_{i}-\delta_{i X}\right) \\
= & \tanh \bar{u}_{i}\left(k_{2}\left(-M \tanh \bar{u}_{i}-M \tanh \rho_{i}+\delta_{i X}\right)+k_{1} \dot{e}_{i X}\right) \\
& -k_{1} \dot{e}_{i X}\left(\tanh \bar{u}_{i}+\tanh \rho_{i}+\frac{\delta_{i X}}{M}\right) \\
= & -k_{2} M \tanh ^{2} \bar{u}_{i}-k_{2} M \tanh \bar{u}_{i} \tanh \rho_{i}-k_{1} \dot{e}_{i X} \tanh \rho_{i} \\
& +k_{2} \tanh \bar{u}_{i} \delta_{i X}-\frac{k_{1}}{M} \dot{e}_{i X} \delta_{i X} \\
\leq & -k_{2} M \tanh \bar{u}_{i}-k_{2} M \tanh \bar{u}_{i} \tanh \rho_{i}-k_{1} \dot{e}_{i X} \tanh \rho_{i} \\
& +\left(k_{2}\left|\tanh \bar{u}_{i}\right|+\frac{k_{1}}{M}\left|\dot{e}_{i X}\right|\right)\left|\delta_{i X}\right|
\end{aligned}
$$

Since the states of a linear system will not diverge to infinite within finite time interval with bounded control input, then, if the initial condition is in the compact set $\left\{\left(e_{i X}\left(t_{0}\right), \dot{e}_{i X}\left(t_{0}\right)\right) \mid \dot{e}_{i X}\left(t_{0}\right)<\infty\right\}$, we obtain that $\dot{e}_{i X}$ is bounded in finite time interval $\left[t_{0}, t_{1}\right)$, where $t_{1}<\infty$. As analyzed in [29], the rotational dynamics of a quadrotor is in the fast time scale $\bar{t}$ with controller (8). Then, there exists a finite time $\bar{t}_{a} \ll t_{1}$ such that the attitude errors $e_{\Theta_{i}}$ enter the neighborhood of the origin, which renders $\left|\delta_{i X}\left(\bar{t}_{a}\right)\right| \leq \zeta$, where $\zeta$ is a scalar. Then, for $t>\bar{t}_{a}$, we have

$$
\begin{aligned}
& \left(k_{2}\left|\tanh \bar{u}_{i}\right|+\frac{k_{1}}{M}\left|\dot{e}_{i X}\right|\right)\left|\delta_{i X}\right| \leq \\
& \left|k_{2} M \tanh ^{2} \bar{u}_{i}+k_{2} M \tanh \bar{u}_{i} \tanh \rho_{i}+k_{1} \dot{e}_{i X} \tanh \rho_{i}\right|
\end{aligned}
$$


Then, $\dot{V}_{i} \leq 0$, when $t>\bar{t}_{a}$. Therefore, the semi-global asymptotic stability of the origin of system 24) is derived, when $t>\bar{t}_{a}$.

Remark 3. In theorem 17 if the condition (25) is satisfied when $\bar{t}_{a}=t_{0}$, the semi-global stability of the system (24) is obtained.

\section{Simulation AND EXPERIMENTAL RESUlts}

In order to illustrate the advantage of the proposed formation controller, we give several simulation and experimental results in this section. In these tests, we consider the formation of four UAVs as shown in Fig 1 b).

\section{A. Simulation results}

In the simulations, we consider a scenario of a formation of four UAVs aggregating to a stationary point.

Note that we use the same attitude and altitude controllers (8), (10) and the gains in all of the tests of comparison in the sequel. We suppose that $g=10 \mathrm{~m} / \mathrm{s}^{2}$. To better illustrate our proposed control, we give a series of simulation results with different formation controllers in table I.

\begin{tabular}{|c|c|c|c|}
\hline No. & Formation controllers & $k_{1}$ & $k_{2}$ \\
\hline 1 & $-4 \tanh \left(\bar{u}_{i}\right)+\ddot{X}_{i}^{d}$ & 0.5 & 0.5 \\
\hline 2 & $-4 \tanh \left(\bar{u}_{i}\right)+\ddot{X}_{i}^{d}$ & 3 & 10 \\
\hline 3 & $-2 \tanh \left(\bar{u}_{i}\right)-2 \tanh \left(\exp ^{-20 e_{i X}^{2}} \dot{e}_{i X}\right)+\ddot{X}_{i}^{d}$ & 0.1 & 0.5 \\
\hline 4 & $-2 \tanh \left(\bar{u}_{i}\right)-2 \tanh \left(5 \exp ^{-20 e_{i X}^{2}} \dot{e}_{i X}\right)+\ddot{X}_{i}^{d}$ & 0.5 & 0.5 \\
\hline 5 & $-4 \tanh \left(0.5 \tanh \left(\bar{u}_{i}\right)+2 \dot{e}_{i X}\right)+\dot{X}_{i}^{d}$ & 10 & 5 \\
\hline 6 & $-2 \tanh \left(\bar{u}_{i}\right)-2 \tanh \left(4.5 \dot{e}_{i X}\right)+\dot{X}_{i}^{d}$ & 8 & 6.5 \\
\hline
\end{tabular}

TABLE I: Formation controllers for comparison

In Table I the controllers 1 and 2 represent bounded PD controllers with different gains, where the hyperbolic tangent functions are used but without CNF. Controllers 3 and 4 are our proposed formation controller in (15), where the CNF is added. The comparison of controllers 1,2, 3 and 4 shows the effects of the CNF and gives an idea of tuning the gains.

Controller 5 and 6 represent two bounded controllers with hyperbolic tangent functions in both nested and added forms. Through the comparison of controllers 5 and 6 with controller 4 , which is proposed in this paper, we can observe the advantage of adding the $\mathrm{CNF}$ in the formation control of quadrotors.

When we use the bounded PD controllers 1 and 2, high gains should be selected in order to obtain a fast rising response. For instance, we can observe from Fig 3 that by augmenting $k_{1}$ the rising response time decreases and by augmenting $k_{2}$ the overshoot is reduced. However, we find that the output curves have small and high-frequency oscillations when high gains in controller 2 are used. In this case, the system will be very sensitive to sensor noise.

The bounded formation control with CNF is given in Fig 4 . We observe that by enhancing the effect of CNF, the overshoot can be reduced, while the response speed keeps rapid. In these formation controllers, we avoid a very high selection of gains $k_{1}$ and $k_{2}$.
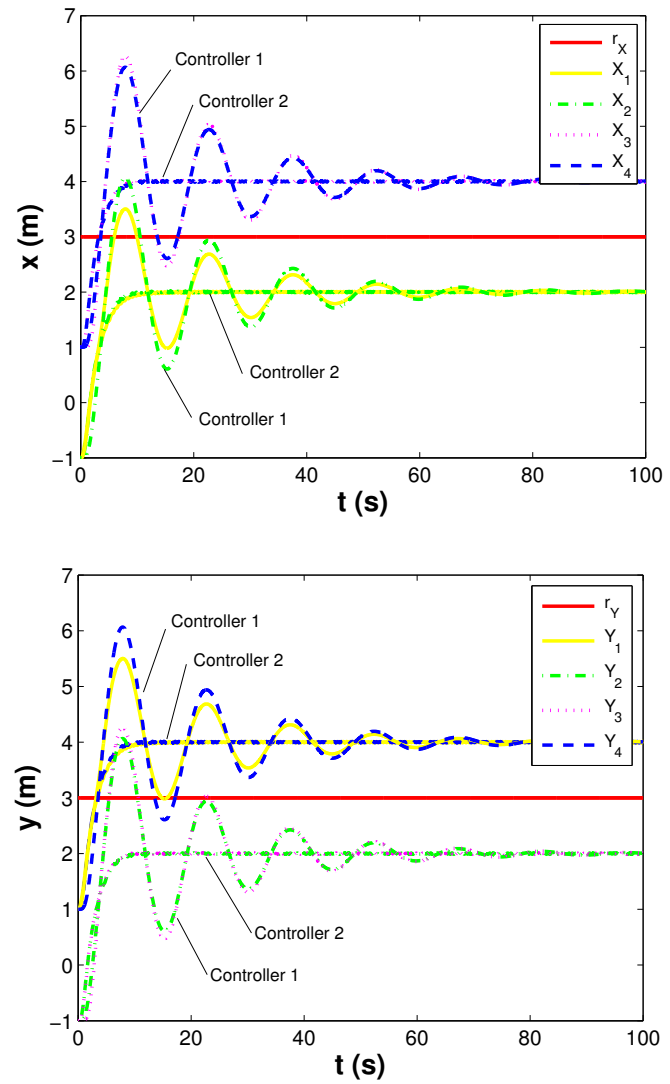

Fig. 3: Bounded PD controllers 1 and 2 with different gains (shown in table I in four UAVs formation control.
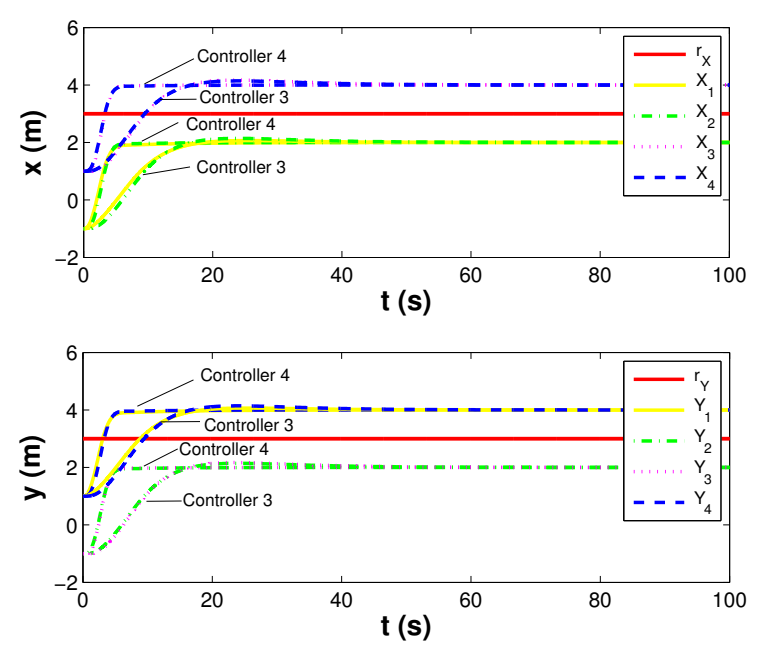

Fig. 4: Formation of four UAVs with controllers 3 and 4 in table I

It is worth to note that the selection of the variable gain $k_{N}$ is not fixed on equation (16). It only needs to satisfy constrains in proposition (5). In this paper, the selection of $k_{N}$ has a physical meaning. We observe that $k_{N}$ is small if the formation error $e_{i X}$ is large such that the rapid rising response is guaranteed. When $e_{i X}$ becomes small, the quadrotor approaches the desired position. Then, $k_{N}$ increases in order to increase the damping of system, such that large overshoot can be avoided.

The comparison of formations with the controller 5 , which 
is in nested form, and our proposed controller (controller 4) is given in Fig. 5. The gains of controller 5 are selected such that the response speed is as fast as controller 4 . We observe that although the response speed of controller 5 is also rapid, the settling time is nevertheless longer and the overshoots are a little bit bigger. Furthermore, we observe that the output of the quadrotors using controller 5 has small and high-frequency oscillations. That is caused by the choice of high gains, which are selected to have a high bandwidth for the system. If we plot the control signals, we can observe that control signals greatly oscillate, as seen in Fig 6 on the top. Then, if we continue increasing the gains for having a bigger bandwidth, the outputs will oscillate more seriously. However, owing to the added CNF, smaller gains in controller 4 can achieve a very satisfactory performance while control signals are much less oscillating than that of controller 5, see Fig 6 on the bottom.
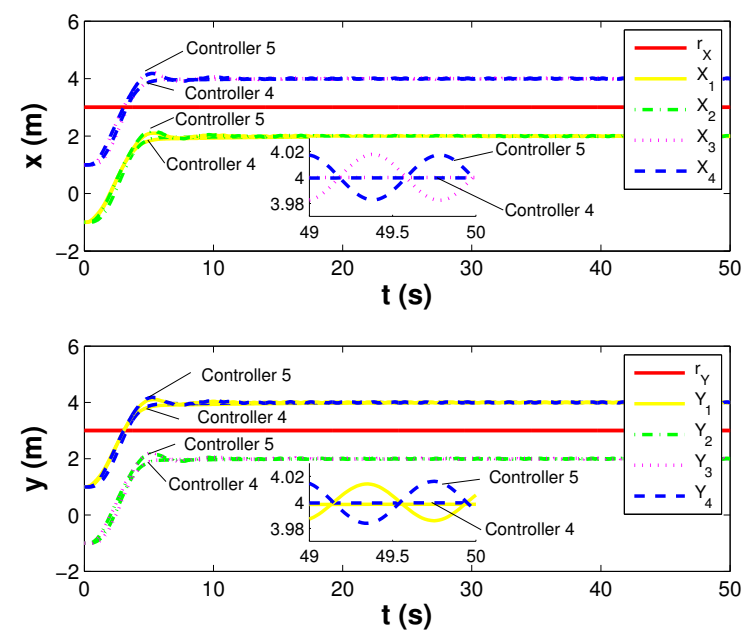

Fig. 5: Comparison of controller 4 and controller 5 used in formation of four UAVs

We also compare the proposed controller 4 with the controller 6 , which is in added form. By tuning the gains of controller 6 , we can also obtain a satisfactory performance such as for controller 4, as shown in Fig.7. However, when we observe their steady states, we see small and high-frequency oscillations in the quadrotors positions using controller 6 .

According to the foregoing simulation results, we can observe that the proposed formation controller performs rapid response and small overshoot at the same time in transient period for multi-UAV formations. Therefore, the proposed controller is suitable for aggressive guidance of quadrotors formations. We will show this aggressive guidance in the following experimental subsection.

\section{B. Experimental results}

Heudiasyc laboratory has developed a PC-based simulatorexperiment framework for controlling a quadrotor and also a formation of quadrotors. It is important to note that within this framework, the control signals are calculated on the UAVs rather than on a $\mathrm{PC}$ in real-time experiments. The quadrotors are Parrot AR Drone 2. Some techniques in the Paparazzi
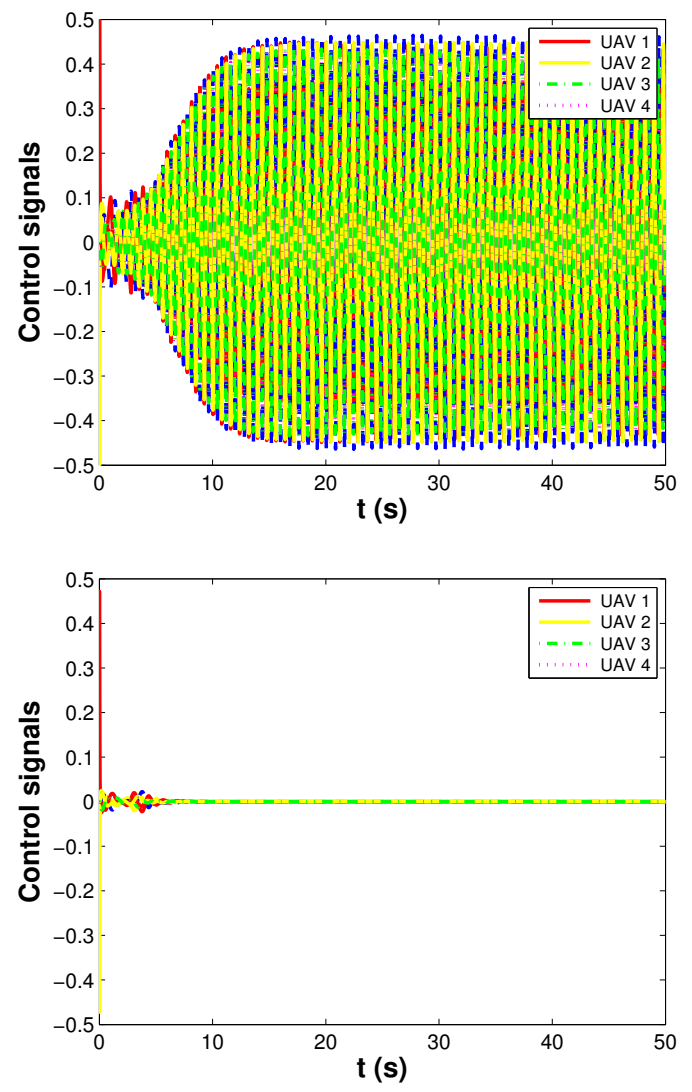

Fig. 6: Control outputs of controller 5 (top) and controller 4 (bottom)
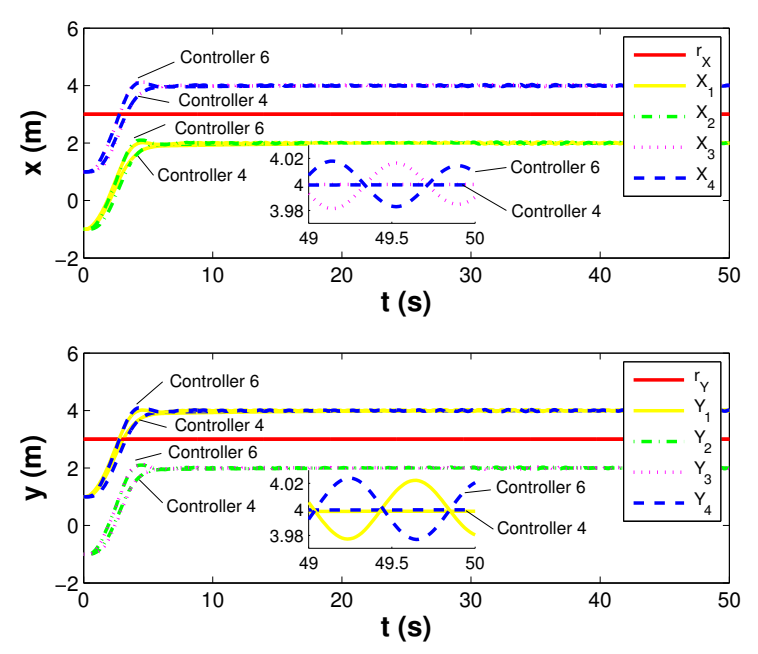

Fig. 7: Comparison of controller 4 and controller 6 used in formation of four UAVs 
Project ${ }^{1}$, such as communication protocol, are applied here in order to implement our algorithms on the drones. We can use the available sensors and the materials of the Parrot drone. We have completely changed the software in the Parrot. We delete the on-board Linux system and implement the Poky 12.0 system. Therefore, we can implement our control algorithm both on low level (such as attitude and altitude control) and high level (such as the formation controller).

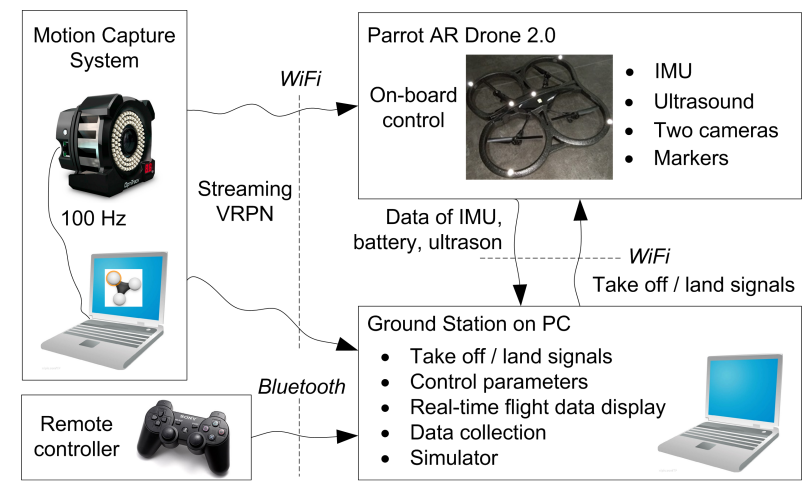

Fig. 8: The experimental setup of the real-time experiments

The experimental setup is shown in Fig 8 In the experiments, the motion capture system Optitrack is used to localize the UAVs in the formation. The system Optitrack collects the positions and attitude angles of the quadrotors and broadcast to all of the quadrotors. Nevertheless, each UAV only uses its neighbors' states (position, velocity) for formation control. Therefore, the formation algorithm is distributed.

The proposed formation controller for multi-quadrotor systems is implemented in the following two tasks. The interacting relation of the UAVs are represented in Fig 1 b.

1) Aggregation to a destination point: We give two experiments of four quadrotors formation with bounded PD controller (i.e. controller 1 in table $\mathrm{I}$ with gains $M=4$, $k_{1}=0.5$, and $k_{2}=0.3$ ) and the proposed formation controller (15) with gains $M=2, k_{1}=0.5, k_{2}=0.1, \eta_{1}=0.4$, and $\eta_{2}=10$. For both controllers, the term $\ddot{X}_{i}^{d}=0$. The outputs curves are shown in Fig 9 and Fig 10 respectively. We note that these two controllers have similar bounds. We observe that in both tests, the quadrotors are able to converge to the desired positions with hyperbolic tangent function-based bounded PD controller. However, after adding CNF, the output curves with the proposed controller has smaller overshoot and more rapid response, as shown in Fig 10

Furthermore, if we plot the inter-distances between quadrotors in Fig 11 and Fig 12 we can observe that the formation pattern is more satisfactorily maintained when the $\mathrm{CNF}$ is added. The corresponding video is available on the site https://www.youtube.com/watch?v=1rO04JkTl5I\&index= 8\&list=PLlJVTLqwkzA8B4ZHPoPH44g0wQmsV2lt_\&t=5s

2) Circular trajectory tracking: The objective is to track a circular trajectory whose radius is $2 \mathrm{~m}$ and the linear velocity is up to $2.7 \mathrm{~m} / \mathrm{s}$, which is the RFT. The gains of controller

1http://wiki.paparazziuav.org/wiki/Main_Page

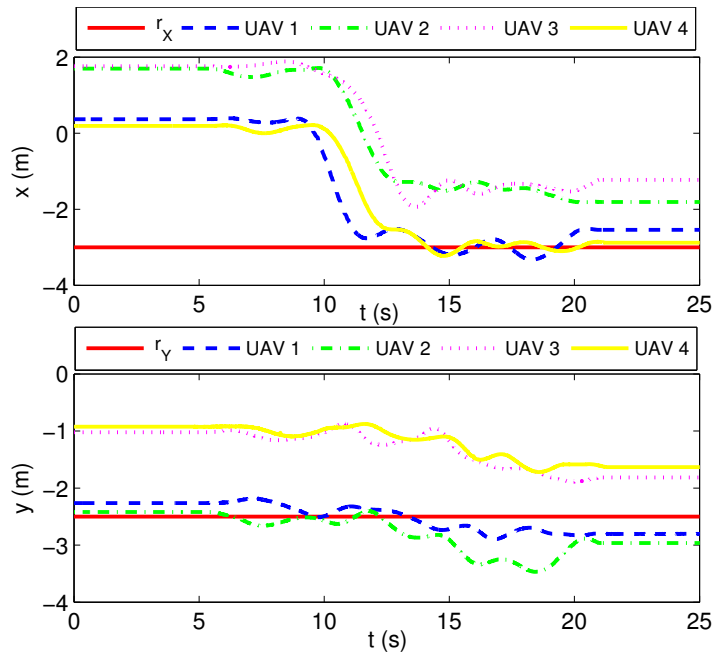

Fig. 9: Real-time experiment: Bounded PD controller in aggregation of four quadrotors formation

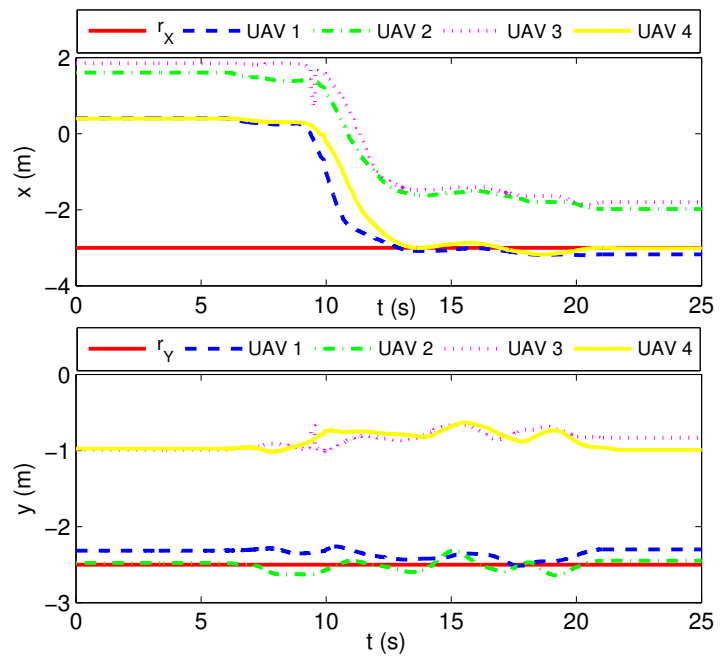

Fig. 10: Real-time experiment: The proposed formation controller in aggregation of four quadrotors formation. (We should note that the curve of UAV 3 (purple-dashed line) has a sharp peak near $10 \mathrm{~s}$ at the beginning of the formation. This is caused by the OptiTrack system, which is perturbed by the light, but not caused by the controller. )

(15) are selected as follows: $M=3, k_{1}=1.5, k_{2}=0.1$, $\eta_{1}=1$, and $\eta_{2}=10$. In this scenario, the group of UAVs has one leader and three followers. Only the leader knows the RFT, while the followers follow the leader or other followers.

The output curves of the four UAVs are shown in Fig.13 We can observe that the outputs of UAV 3, which has no interaction with UAV 1 (the leader), have very small delay with respect to the leader. According to Fig 14, the pitch and roll angles of the four UAVs are up to 20 degrees. The UAVs keep a formation pattern and perform an aggressive guidance, according to [25]. The corresponding video is available on https://www.youtube.com/watch?v=639z0GLb8cM\&index= 


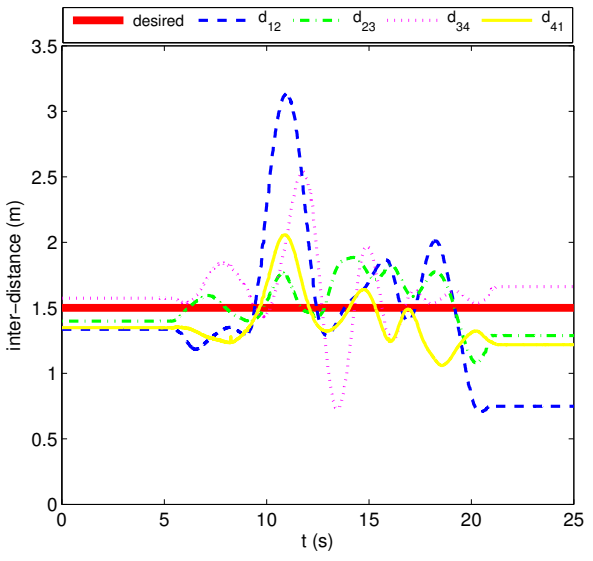

Fig. 11: Real-time experiment: The inter-distances of quadrotors with bounded PD controller in aggregation of four quadrotors formation

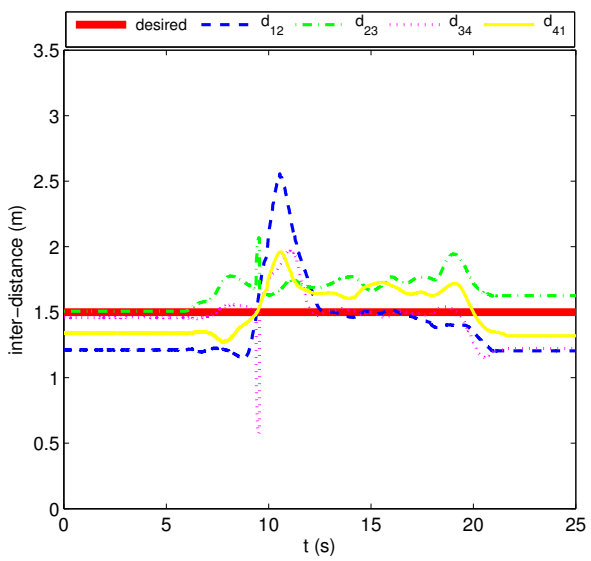

Fig. 12: Real-time experiment: The inter-distances of quadrotors with proposed formation controller in aggregation of four quadrotors formation. Note that the sharp peaks on $d_{23}$ and $d_{34}$ near $10 s$ are caused by the instantaneous wrong location of UAV 3 from OptiTrack system.

\section{9\&list=PLIJVTLqwkzA8B4ZHPoPH44g0wQmsV2lt_\&t=5s}

\section{CONClusion}

In this paper, the interaction matrix is proposed to describe the "interaction relations" in a leader-follower multi-agent formation. The hyperbolic tangent-based bounded control with composite nonlinear feedback is developed for quadrotors formation control. By using this controller, the performance of the formation is improved, which is illustrated by simulation results. The proposed formation controllers are also validated by real-time experiments, where the aggressive guidance of four quadrotors formation is realized.
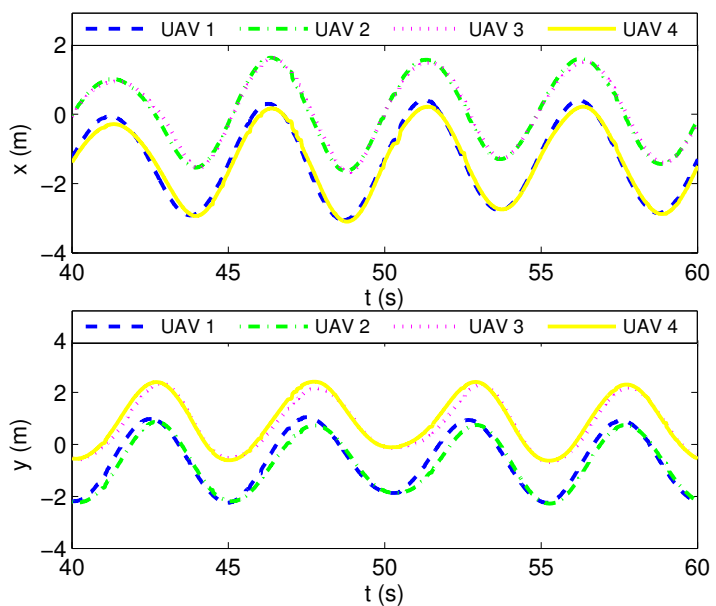

Fig. 13: Real-time experiment: guidance of four UAVs. The RFT is a circle. The delay between UAV 1 (the leader) and UAV 3 (the follower without interaction to the leader) is very small.
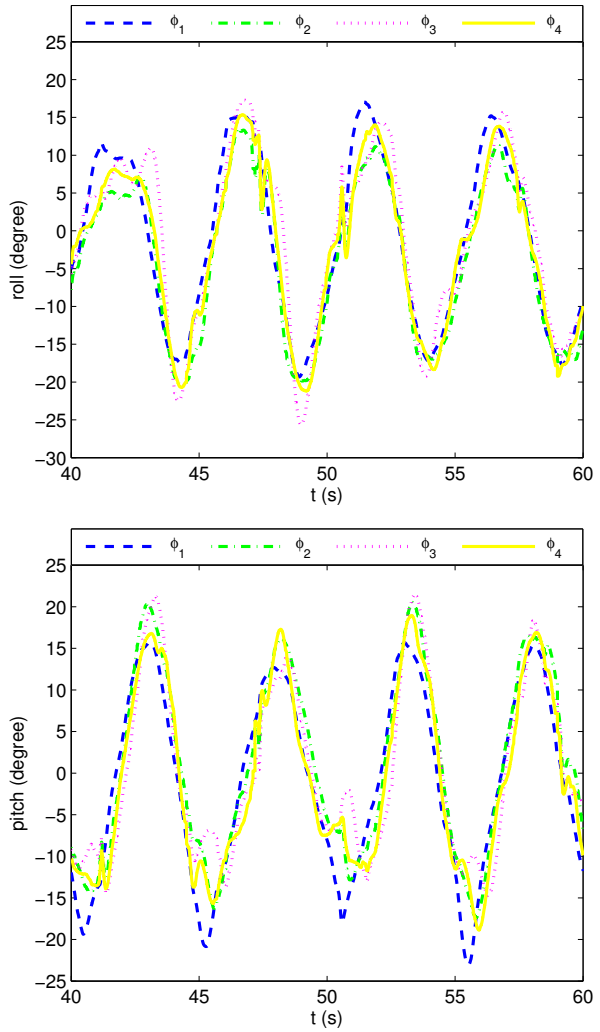

Fig. 14: Real-time experiment: guidance of four UAVs. The RFT is a circle. The roll angles of the four UAVs are up to 20 degrees.

\section{REFERENCES}

[1] E. Semsar-Kazerooni and K. Khorasani, "Optimal consensus algorithms for cooperative team of agents subject to partial information," Automatica, vol. 44, no. 11, pp. 2766 - 2777, 2008. [Online]. Available: http://www.sciencedirect.com/science/article/pii/S0005109808002719

[2] M. Dogar, A. Spielberg, S. Baker, and D. Rus, "Multi-robot grasp planning for sequential assembly operations," in 2015 IEEE International 
Conference on Robotics and Automation (ICRA), May 2015, pp. 193200.

[3] W. Ren, R. Beard, and E. Atkins, "Information consensus in multivehicle cooperative control: collective group behavior through local interaction," IEEE Control Systems, vol. 27, no. 2, pp. 71-82, April 2007.

[4] K. Sreenath and V. Kumar, "Dynamics, control and planning for cooperative manipulation of payloads suspended by cables from multiple quadrotor robots," in Proceedings of Robotics: Science and Systems, Berlin, Germany, June 2013.

[5] A. Franchi, C. Secchi, M. Ryll, H. Bülthoff, and P. R. Giordano, "Shared control: Balancing autonomy and human assistance with a group of quadrotor uavs." IEEE Robotics and Automation Magazine, Special Issue on Aerial Robotics and the Quadrotor Platform, vol. 19, pp. 57-68, September 2012.

[6] R. Ritz and R. D'Andrea, "Carrying a flexible payload with multiple flying vehicles," in 2013 IEEE/RSJ International Conference on Intelligent Robots and Systems (IROS), Nov 2013, pp. 3465-3471.

[7] B. Grocholsky, V. Kumar, and H. Durrant-Whyte, "Anonymous cooperation in robotic sensor networks," in American Association for Articial Intelligence, 2004

[8] R. Saber and R. Murray, "Consensus protocols for networks of dynamic agents," in Proceedings of the 2003 American Control Conference, vol. 2, June 2003, pp. 951-956.

[9] A. Franchi and P. R. Giordano, "Online leader selection for improved collective tracking and formation maintenance," IEEE Transactions on Control of Network Systems, 2016. [Online]. Available: http://arxiv.org/abs/1305.5719

[10] P. Robuffo Giordano, A. Franchi, C. Seccos, and H. Bulthoff, "A passivity-based decentralized strategy for generalized connectivity maintenance," The International Journal of Robotics Research, vol. 32, no. 3, pp. 229-323, 2016. [Online]. Available: http: //arxiv.org/abs/1305.5719

[11] Q. Jiang and V. Kumar, "The inverse kinematics of cooperative transport with multiple aerial robots," IEEE Transactions on Robotics, vol. 29, no. 1, pp. 136-145, Feb 2013.

[12] A. Kushleyev, V. Kumar, and D. Mellinger, "Towards a swarm of agile micro quadrotors," in Proceedings of Robotics: Science and Systems, Sydney, Australia, July 2012.

[13] N. Michael, J. Fink, and V. Kumar, "Cooperative manipulation and transportation with aerial robots," in Proceedings of Robotics: Science and Systems, Seattle, USA, June 2009.

[14] P. Dames and V. Kumar, "Autonomous localization of an unknown number of targets without data association using teams of mobile sensors," IEEE Transactions on Automation Science and Engineering, vol. 12, no. 3, pp. 850-864, 2015.

[15] M. Saska, J. Chudoba, L. Precil, J. Thomas, G. Loianno, A. Tresnak, V. Vonasek, and V. Kumar, "Autonomous deployment of swarms of micro-aerial vehicles in cooperative surveillance," in International Conference on Unmanned Aircraft Systems (ICUAS), May 2014, pp. 584595.

[16] D. Panagou and V. Kumar, "Cooperative visibility maintenance for leader-follower formations in obstacle environments," IEEE Transactions on Robotics, vol. 30, no. 4, pp. 831-844, Aug 2014.

[17] M. Turpin, N. Michael, and V. Kumar, "Trajectory design and control for aggressive formation flight with quadrotors," Autonomous Robots, vol. 33, no. 1-2, pp. 143-156, 2012. [Online]. Available: http://dx.doi.org/10.1007/s10514-012-9279-y

[18] F. Augugliaro, S. Lupashin, M. Hamer, C. Male, M. Hehn, M. W. Mueller, J. S. Willmann, F. Gramazio, M. Kohler, and R. D'Andrea, "The flight assembled architecture installation: Cooperative construction with flying machines," IEEE Control Systems, vol. 34, no. 4, pp. 46-64, Aug 2014

[19] D. López-Araujo, A. Zavala-Río, I. Fantoni, S. Salazar, and R. Lozano, "Global stabilisation of the PVTOL aircraft with lateral force coupling and bounded inputs," International Journal of Control, vol. 83, no. 7, pp. 1427-1441, 2010. [Online]. Available: http://dx.doi.org/10.1080/00207171003758778

[20] N. Cao and A. Lynch, "Inner-outer loop control with constraints for rotary-wing uavs," in International Conference on Unmanned Aircraft Systems (ICUAS), June 2015, pp. 294-302.

[21] M. Hehn, "Quadrocopter trajectory generation and control," in In IFAC World Congress, 2011, pp. 1485-1491.

[22] H. Su, M. Chen, J. Lam, and Z. Lin, "Semi-global leader-following consensus of linear multi-agent systems with input saturation via low gain feedback," IEEE Transactions on Circuits and Systems I: Regular Papers, 2013.
[23] Z. Lin, M. Pachter, and S. Banda, "Toward improvement of tracking performance nonlinear feedback for linear systems," International Journal of Control, vol. 70, no. 1, pp. 1-11, 1998. [Online]. Available: http://dx.doi.org/10.1080/002071798222433

[24] B. Chen, T. Lee, K. Peng, and V. Venkataramanan, "Composite nonlinear feedback control for linear systems with input saturation: theory and an application," IEEE Transactions on Automatic Control, vol. 48, no. 3, pp. 427-439, Mar 2003.

[25] H. Huang, G. Hoffmann, S. Waslander, and C. Tomlin, "Aerodynamics and control of autonomous quadrotor helicopters in aggressive maneuvering," in IEEE International Conference on Robotics and Automation (ICRA), May 2009, pp. 3277-3282.

[26] R. Olfati-Saber, J. Fax, and R. Murray, "Consensus and cooperation in networked multi-agent systems," Proceedings of the IEEE, vol. 95, no. 1, pp. 215-233, Jan 2007.

[27] L. Hogben, Handbook of Linear Algebra, 1st ed. CRC Press, 2007.

[28] W. Ni and D. Cheng, "Leader-following consensus of multi-agent systems under fixed and switching topologies," Systems \& Control Letters, vol. 59, no. 3-4, pp. 209-217, 2010.

[29] Z. Hou and I. Fantoni, "Composite nonlinear feedback-based bounded formation control of multi-quadrotor systems," in 15th European Control Conference (ECC16), Aalborg, Denmark, Jun, 2016.

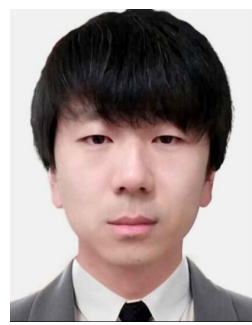

Zhicheng Hou received the Ph.D degree in control engineering from the Université de Technologie de Compiègne, Compiègne, France in 2016. Now, he is an assistant researcher in Guangzhou Institute of Advanced Technology, Chinese Academy of Science in China.

His current research interests include nonlinear control and multi-robot system.

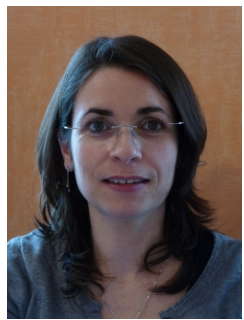

Isabelle Fantoni received the $\mathrm{PhD}$ degree, with the European label, in Non-linear Control for Underactuated Mechanical Systems, in 2000 from the Université de Technologie de Compiègne, in France. Since October 2001, she is a permanent Researcher at Heudiasyc laboratory, UTC, in Compigne, France, employed by the French National Foundation for Scientific Research (CNRS) and CNRS Research Director since October 2013. Her research interests include non-linear control, modelling and control for Unmanned Aerial Vehicles (UAVs), fault-tolerant control for UAVs, vision for navigation of aerial vehicles, cooperation of UAVs, heterogeneous robotic systems in cooperation. 